\title{
THE PREPARATION OF THE CRYOMAGNETS AND THE ASSEMBLY OF THE LHC TEST STRING 2
}

\author{
O.Andujar, D.Bozzini, C.Calzas-Rodriguez, P.Cruikshank, O.Desebe, T.Dobers, A.Jacquemod, \\ N.Kos, P.Lepeule, W.Maan, D.Missiaen, V.Parma, G.Riddone, F.Rodriguez-Mateos, P.Rohmig, \\ R.Saban, G.Schneider, L.Serio, B.Skoczen, JP.Tock, R.Veness, Ch.Vuitton, \\ CERN, Geneva, Switzerland
}

\begin{abstract}
The numerous complex activities required to prepare the cryomagnets for the installation in String 2 are described. These include the configuration of the mechanical interfaces, the conditioning of the beam tubes, the installation of beam screens and the instrumentation as well as the final checks. The preparation of the cryomagnets for String 2 has been a dress rehearsal for the preparation that the cryomagnets will undergo before their installation in the tunnel. After a description of the interconnection procedures of the components for String 2 , the tests carried-out to release the String for operation are described. A brief account of the lessons learnt is also given.
\end{abstract}

\section{STRING 2}

As for its predecessor, String $2[1,2]$ is built to validate individually the different systems and investigate their collective behaviour in conditions equivalent to those in the machine. String 2 is being assembled in two phases. The facility which will be commissioned during the first phase comprises one half-cell and an additional short straight section. It contains exclusively full-scale prototype magnets heavily instrumented for the experimental programme. The commissioning started at the end of April 2001. Phase 2 of String 2 will include, in addition, three pre-series dipole magnets and will be commissioned in early 2002.

\subsection{Layout}

String 2 consists of two consecutive LHC half-cells terminated, on the upstream end, by the electrical feedbox (DFBS) and, on the downstream end, by the magnet return box (MRB). Each half-cell is composed of one short straight section (SSS) and three 15-m dipoles (MB). The DFBS is a prototype of the arc feed boxes, the DFBAs, and contains the current leads through which the 11 of the 15 electrical circuits are fed. The MRB contains the short circuits and the connection to the cryogenic distribution line (QRL) simulating the jumper connection of the following cell. The QRL distributes and recovers cryogenic helium at various temperatures.

With respect to vacuum, cryogenics, interlocks, protection and powering, Phase 2 of String 2 represents a full cell in the regular part of the LHC arc.

\section{THE PREPARATION OF THE SITE}

String 2 is installed in hall 2173 (SM18) and just fits in the length of the building $(120 \mathrm{~m})$. Because it comprises the cryoline an artificial difference in level was required to simulate the trench in the tunnel. This was achieved using prefabricated concrete slabs.

The interface between the jacks and the floor of the hall was verified by simulating the forces in play when the vacuum vessel is evacuated. A $70 \mathrm{kN}$ force is exerted on the two end faces of the String and are transferred to the floor via the supports of the DFBS and the MRB.

The preparation of the site also included the pulling of the cables and the installation of the crates. All the crates which will be present in the tunnel were installed under the cryostats and those dedicated to the experiments were installed behind the QRL. Although largely dominated by the String specific cabling, $18 \mathrm{~km}$ of cables and the associated connectors for the process control and the data acquisition were pre-installed.

The power converters for the 15 separate circuits, the energy extraction system for the dipole circuit and the lattice sextupoles were installed in the powering area situated at the north end of the String. A kicker power supply and an associated dummy load is foreseen in this area to study the electromagnetic compatibility of converters, front-end electronics, PLCs, etc. All the infrastructure particular to powering was also installed. It includes demineralized water distribution, water and air cooled DC cables and a set of switches to modify the topology of the dipole circuit.

\section{THE PREPARATION OF THE COMPONENTS}

The five magnets installed for Phase 1 underwent extensive tests on dedicated test benches [3]. One of the dipoles was quenched 86 times at different current levels. On one occasion, in this same magnet, the heaters failed to fire because of a short in an instrumentation feedthrough connection box.

Every magnet had to be converted from a test bench configuration to a tunnel compatible configuration. As an example, the upstream end of a dipole is shown before and after the modification (Figure 1 and 2).

Most of the modifications concerned the cryomagnet extremities; in particular the V1 and V2 tubes (the beam 
tubes) which were fitted with beam screens and RF contacts but also the M1, M2 and M3 tubes which contain the bus-bars for the main magnets (the dipole and the two quadrupole circuits) and spool-piece correctors in the dipoles. A final design instrumentation feedthrough and the by-pass diode were mounted on every dipole. Line N, which contains the bus-bar cable for the lattice correctors in the SSS, was mounted and fitted with its flexible hose.

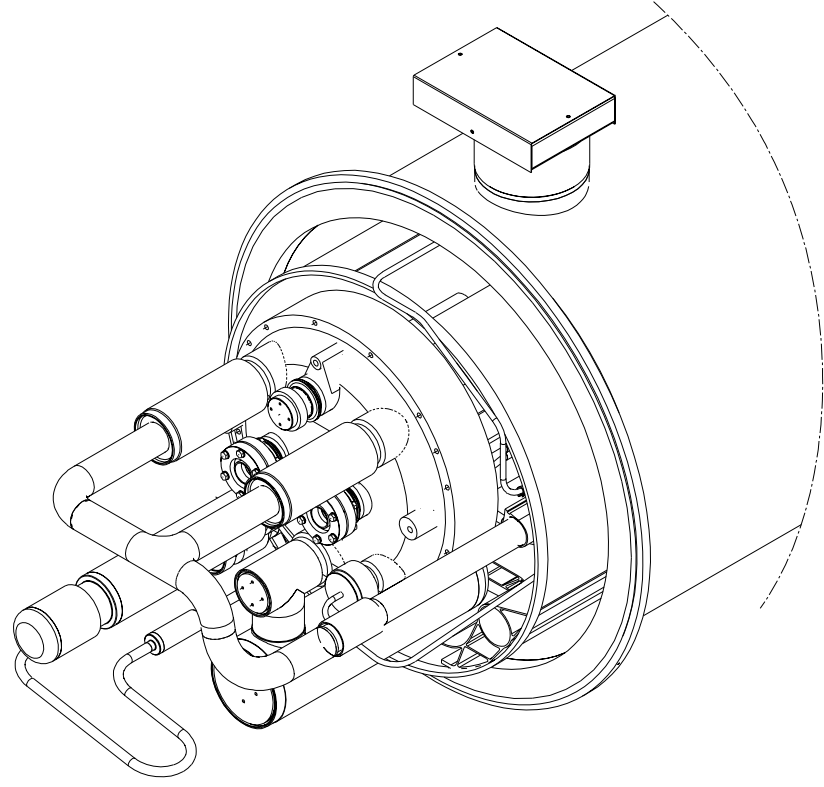

Figure 1: Dipole upstream end in test bench configuration

Although the learning process is clearly visible in the decreasing preparation times, it remains still above the times foreseen for the series magnets.

During their preparation, a number of tests were made to qualify the magnet on reception, to verify the result of an operation and finally qualify the magnet for installation. These tests included :

- the dimensional verification of the magnet extremities,

- the measurement of the cold mass position with respect to fiducials on the vacuum envelope,

- leak tests of all components before installation and after the welds,

- electrical insulation tests of the coils and the instrumentation at the reception and after the installation of the by-pass diode and the instrumentation feedthrough,

- electrical measurements of the transfer function, of the coil resistance and of the continuity of the circuit before installation.

\section{THE ASSEMBLY}

Before the assembly of the cryo-magnets started, the QRL was installed on its supports, welded, leak tested and connected to the cryogenic feed and return boxes. The cryo-magnets were then joined by operations first performed in the innermost tubes (the beam tubes) and then advancing towards the periphery.

After the beam tubes were fitted with the RF contacts and welded, the main bus-bars were soldered and insulated. These were then followed by the ultrasonic welding of the spool-piece bus-bars and the welds of tubes containing the bus-bars. An electrical insulation test was performed after soldering or ultrasonic welding of bus-bars. Although these tests yield no information on the quality of the junction, at least for the ultrasonic welds, a prototype automatic machine with well controlled parameters was used. The quality of these junctions was verified on samples.

The flexible cooling end tubes of the beam-screens and the lines thermalising the support posts and the thermal shield were then welded. After all these welds, a partial leak test was performed.

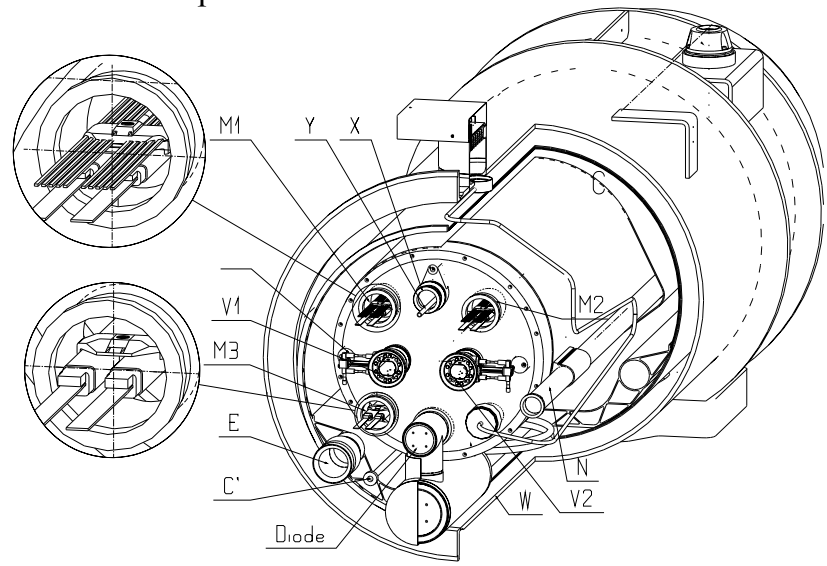

Figure 2 : Dipole upstream end in tunnel configuration

Line $\mathrm{N}$ was not welded until the bus-bar cable was inserted over the full length of the half-cell.

The learning process is also visible for the assembly operation. It is now expected that a typical dipole-dipole interconnect will take 70 hours and the assembly of a full cell in the tunnel will take 700 hours [4].

\subsection{Incidents}

A number of incidents occurred during the preparation and assembly process. Only the most important ones, in terms of consequence they had or could have had, are reported.

A leak in one of the cooling tubes of a beam screen developed after it was inserted in the beam tube and pressure tested. The beam screen was removed and sliced to investigate the leak. Following an extensive analysis, it was concluded that the leak had most likely been caused by remaining traces of chlorine contained in lubricants used during the manufacturing process of the cooling tubes. As a consequence of this incident, the beam screens were extensively tested after each operation during the assembly in the beam tube.

After the measurements on the test bench, the cryomagnets were flushed with high pressure nitrogen gas to expel eventual debris left from manufacturing or 
produced during the tests. This process revealed that the flux loops and the beam tube insulation did not survive a quench: the former were abandoned while the latter was re-designed. A side effect of this process was the blowing of the wires for the test bench specific instrumentation deep inside the domed end of the dipoles. For two dipole magnets these instrumentation wires were left inside the cold mass without being insulated. During the qualification test prior to installation, the value of the coil resistance was found to be unusually low. This was caused by contacts of voltage tap instrumentation wires connected to different parts of the coils. A visual inspection of the bus-bar tubes revealed the presence of the uninsulated wires deep inside the cold mass.

When the last element of the magnet string, SSS3 was installed, the connectors to instrumentation in the vacuum vessel fixed on a flange on the side of the vessel were found to interfere with one of the valve boxes of the QRL. Fortunately, the wires conveyed signals from sensors installed upstream of the vacuum barrier and therefore the set of connectors could be moved to another opening on the vacuum vessel.

\subsection{Non-Conformities}

The following major non-conformities were encountered. They are classified according to their nature: Mechanical

- $\quad$ ovalisation of beam tube in one of the dipoles

- incorrect beam tube length in the second SSS

- incompatibility between the QRL portion and the corresponding SSS portion of the jumper connection

- leaks of the bus-bar plugs in the first SSS

- incorrect position of spool piece corrector bus-bars in SSSs

\section{Electrical}

- short to ground in an orbit corrector of the second SSS

- damage to orbit corrector current leads in the first SSS

- short to ground in one sextupole spool-piece corrector in one dipole

Instrumentation related

- missing voltage taps in second SSS

- broken pressure sensor in first SSS

All the non-conformities were either resolved by repairing the fault, or by by-passing the faulty component or else leaving the component as is.

\subsection{Quality Assurance}

The Quality Assurance requirements were applied to interfaces and components, assembly technologies and assembly procedures. The relevant control/inspection procedures and check lists were developed and tested. Finally, one Manufacturing and Test Folder (MTF) per interconnect was prepared: the document contains all the parameters and data related to the components of the interconnections and the relevant assembly technologies.

\section{LESSONS LEARNT}

In the assembly phase, String 2 has already yielded precious information about the mechanical design of interfaces, alignment, assembly and quality assurance procedures. It has also validated the assembly process. The presence for the first time of components like beam screens, RF contacts in the interconnects, BPMs in the short straight sections has validated the design and highlighted possible improvements for the series production of the magnets. The assembly and the commissioning [5] included also components like the DFB, magnet and cryogenic return boxes which are peculiar to the String but are also prototypes for LHC.

A number of points can be improved for the next phase of the assembly of String 2. Namely, the definition of responsibility in border areas (e.g. installation of the diode), the definition of procedures and their sequencing (e.g. electrical tests), the optimisation of the procedures (e.g. bus-bar soldering, ultrasonic welding \& insulation). Experience gained during this first phase will certainly lead to improved tooling.

Last but not least, a work flow diagram for the preparation and assembly work which integrates the full process across group boundaries was missing. It would have improved performance and resulted in less work and shorter times. The MTF for each component with the history of the component before its delivery for installation in String 2 was either incomplete or missing. For Phase 2, emphasis should be put on this document so that all the operations and tests carried-out during the assembly are also recorded in a single document.

\section{ACKNOWLEDGEMENTS}

The quality of the preparation achieved despite the incidents and the non-conformities enumerated above, was remarkable in as much as, many parameters were measured to be well within tolerance of what is aimed for the collider. This was possible thanks to the quality of the studies, preparation and assembly work of many colleagues in the Accelerator and Technical Sectors of CERN.

\section{REFERENCES}

[1] F. Bordry et al., The LHC Test String 2 : Design Study, LHC Project Report, March 1998

[2] F. Bordry et al., The LHC Magnet String Programme: Status and Future Plans, PAC'99, New-York, April 1999

[3] L.Bottura et al., Performance of the LHC Final Design Full Scale Superconducting Dipole Prototypes, IEEE Trans. on Appl. Sup., vol. 11 No 1, pp.1554-1557, 2001

[4] J.Ph.Tock, Cryomagnets interconnections and connections to the cryogenic distribution line, LHC Days, Villars 2001

[5] F. Bordry et al., The commissioning of the LHC Test String 2, this conference. 